PAPER • OPEN ACCESS

\title{
Production of ibuprofen-magnetite nanocomposites by pulsed laser ablation
}

To cite this article: Tamás Gera et al 2021 J. Phys. D: Appl. Phys. 54395401

View the article online for updates and enhancements.

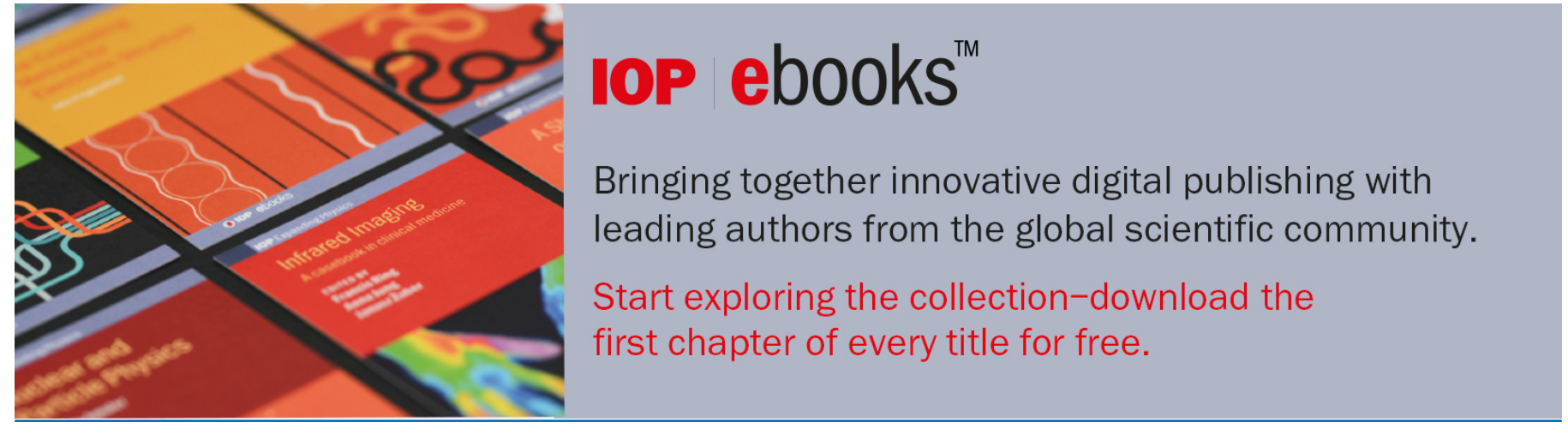

This content was downloaded from IP address 160.114 .23 .240 on 28/09/2021 at 13:55 


\title{
Production of ibuprofen-magnetite nanocomposites by pulsed laser ablation
}

\author{
Tamás Gera ${ }^{1, *}\left(\mathbb{D}\right.$, Tomi Smausz ${ }^{1}$, Tibor Ajtai ${ }^{1}$, Boldizsár Kurilla ${ }^{1}$, Zsolt Homik ${ }^{1}$, \\ Judit Kopniczky ${ }^{1}$, Zoltán Bozóki ${ }^{1}{ }^{10}$, Piroska Szabó-Révész ${ }^{2}$, Rita Ambrus ${ }^{2}$ \\ and Béla Hopp ${ }^{1,3}$ (1)

\footnotetext{
${ }^{1}$ Department of Optics and Quantum Electronics, University of Szeged, Dóm tér 9, 6720 Szeged, Hungary

${ }^{2}$ Institute of Pharmaceutical Technology and Regulatory Affairs, University of Szeged, Eötvös utca 6, 6720 Szeged, Hungary

${ }^{3}$ Department of Materials Science, Interdisciplinary Excellence Centre, University of Szeged, Dugonics tér 13, 6720 Szeged, Hungary
} \\ E-mail: gertamc2@gmail.com
}

Received 26 March 2021, revised 14 May 2021

Accepted for publication 3 June 2021

Published 19 July 2021

\begin{abstract}
We investigated the application of pulsed laser ablation (PLA) for the production of magnetic nanoparticles (MNPs)/drug nanocomposites. Magnetic drug delivery vehicles can provide a multifunctional theranostic platform and allow for a combination of diagnostics, monitoring and therapeutics. We applied nanosecond laser pulses at two different wavelengths (Nd:YAG laser, $\lambda_{1}=532 \mathrm{~nm} / \lambda_{2}=1064 \mathrm{~nm}, \mathrm{FWHM}=6 \mathrm{~ns}$ ) for the ablation of target tablets consisting of a mixture of ibuprofen (as a model drug) and magnetite NPs in various mass ratios.

Fourier-transform infrared and Raman spectroscopy investigations of the ablated particles revealed that PLA induced no chemical changes in the pharmaceutical compound. The size distributions of the composite particles were established using a particle size analyzer (scanning mobility particle sizer), and 100-150 nm mean particle size values were obtained. SEM, energy dispersive $\mathrm{x}$-ray spectroscopy and fast photography studies provided morphological and compositional information about the ablated particles. We demonstrated that PLA offers a simple and chemical free method for producing MNPs/drug nanocomposites, and it can be applied for the future development of new theranostic nanosystems.
\end{abstract}

Keywords: nanocomposites, drug preformulation, pulsed laser ablation, magnetite nanoparticle, theranostic, drug delivery

(Some figures may appear in color only in the online journal)

* Author to whom any correspondence should be addressed. 


\section{Introduction}

Nanomedicine, an emerging field of medicine, shows great promise for developing innovative nanotechnological solutions. With the help of nanocomposites it is now possible to diagnose and/or treat several diseases at a molecular level [1]. Magnetosensitive nanocomposites as drug delivery systems can induce the accumulation and then the release of pharmaceuticals at specific body sites to improve their therapeutic effect while minimizing adverse reactions, and in combination with the MRI technique, the path of nanocomposites can be traced in the body [2-9].

The core of magnetic drug delivery vehicles is commonly magnetite $\left(\mathrm{Fe}_{3} \mathrm{O}_{4}\right)$ due to its superparamagnetic properties and good biocompatibility [10-14]. The applicability of magnetic nanoparticles (MNPs) in therapy and diagnostics strongly depends on the particles' size, shape, surface properties and terminal functional groups [15]. Thus, the production of drugloaded MNPs is of great importance, but it often requires complex and costly technology [16]. Therefore, the search for novel methods which may simplify the preparation of magnetosensitive nanocomposites is still relevant.

Pulsed laser ablation (PLA) is a well-established and efficient method for the nanonization of both organic and inorganic materials. In the course of PLA, a high-energy pulsed laser beam is focused onto the target. This creates a microexplosion at the surface region and particle ejection occurs $[17,18]$. It has already been shown that even drug particles can be ground by PLA with no chemical decomposition of the initial compounds [18-22]. Moreover, we previously reported the successful size reduction of ibuprofen (and other nonsteroidal anti-inflammatory drug (NSAID)) particles with the same method [23]. In the present experiment we applied PLA for loading the magnetite NPs with ibuprofen as a model compound. Due to its low solubility, ibuprofen can serve as a functionalization molecule for surface modification or as a linker for targeted drug delivery.

By the laser ablation of ibuprofen and magnetite NP mixtures, we could utilize the size reduction ability of PLA in combination with the production of composite particles. The fluence of the Nd:YAG laser system $\left(\lambda_{1}=1064 \mathrm{~nm}\right.$, $\lambda_{2}=532 \mathrm{~nm}$ ) was adjusted to gain the highest yield of ablated particles without any chemical decomposition of ibuprofen [23]. We investigated the chemical composition and the size distribution of the ablated particles as well as their behavior in an external magnetic field.

Our motivation was to show that PLA has a favorable and multifunctional potential in the development of new pharmaceutical formulations in terms of both particle size reduction and the encapsulation of MNPs. Our aim was to produce a magnetite based nanocomposite in which ibuprofen as a model drug is merged with the MNPs. PLA can give a new and 'green' approach for the generation of drug loaded magnetic nanocomposites. Here we present a relatively simple, singlestep approach to provide magnetically responsive nanocomposite carrier systems for drugs. Our method could provide a new approach for the development of drug loading through direct encapsulation of MNPs and therapeutic agents [24].

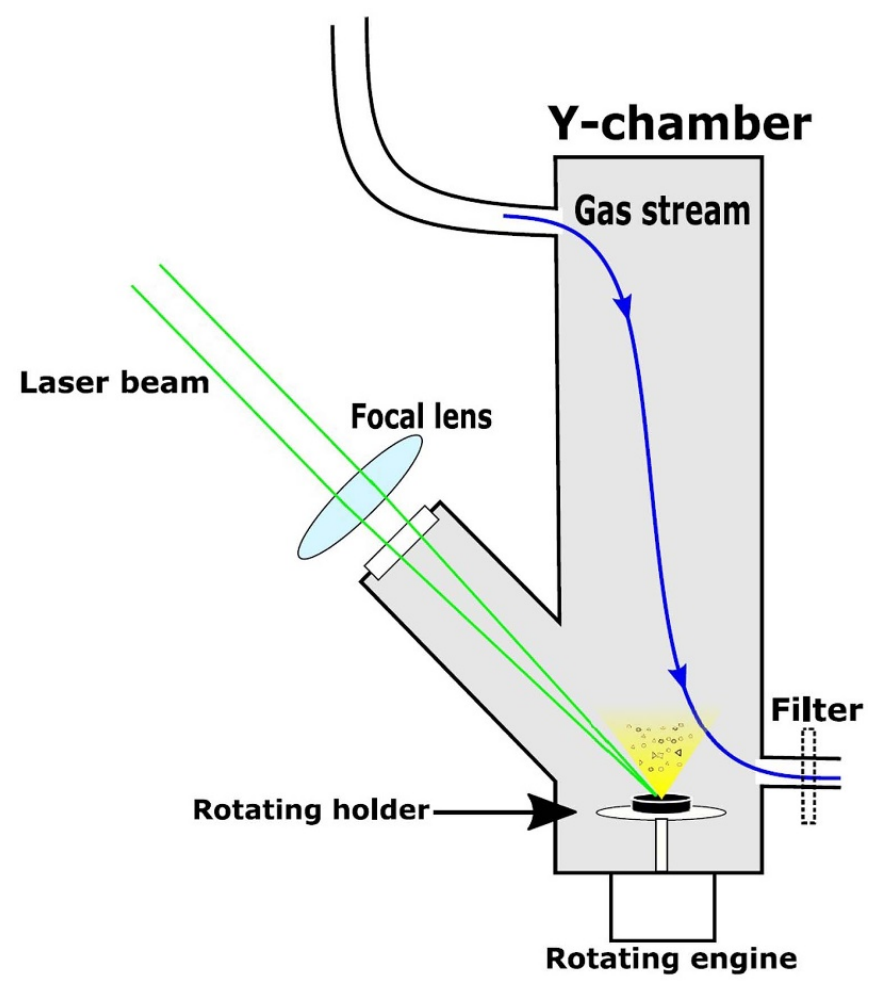

Figure 1. Experimental setup for PLA of mixed tablets.

\section{Materials}

\subsection{Ibuprofen}

Ibuprofen ( $\alpha$-methyl-4-(isobutyl) phenylacetic acid) was obtained from Sigma-Aldrich Ltd, (Saint Louis, Missouri, USA) $(\geqslant 98 \%)$. It is a white powder with a particle size of $15.3 \mu \mathrm{m}(d(0.5))$. NSAID classification: propionic acid derivatives. Thermal properties: melting point $T_{\mathrm{m}}=75^{\circ} \mathrm{C}-$ $77^{\circ} \mathrm{C}$; boiling point $T_{\mathrm{b}}=157^{\circ} \mathrm{C}$; decomposition temperature $T_{\text {dec }}=230{ }^{\circ} \mathrm{C}-250{ }^{\circ} \mathrm{C}[25]$.

\subsection{Magnetite nanopowder}

Magnetite $\left(\mathrm{Fe}_{3} \mathrm{O}_{4}\right)$ was acquired from Sigma-Aldrich Ltd, (Saint Louis, Missouri, USA) ( $\geqslant 97 \%$ trace metal basis). It is a black nanopowder with a particle size of $50-100 \mathrm{~nm}$. Thermal properties: melting point $T_{\mathrm{m}}=1597^{\circ} \mathrm{C}$; boiling point $T_{\mathrm{b}}=2623{ }^{\circ} \mathrm{C}[26]$.

\section{Experimental methods}

\subsection{PLA of ibuprofen-magnetite composite tablets}

We prepared ibuprofen-magnetite composite tablets with $4: 1$; 3:1 and 2:1 (ibuprofen:magnetite) mass ratios. The component powders were mixed and then compacted by a hydraulic compactor at $175 \mathrm{MPa}$ pressure to form the target samples. During the PLA of the tablets we applied two laser wavelengths: the first and second harmonics of an Nd:YAG laser (Quantel, $\mathrm{FWHM}=6 \mathrm{~ns}, \lambda=532 \mathrm{~nm} / 1064 \mathrm{~nm}, f=1 \mathrm{~Hz}$ ) with a fluence 


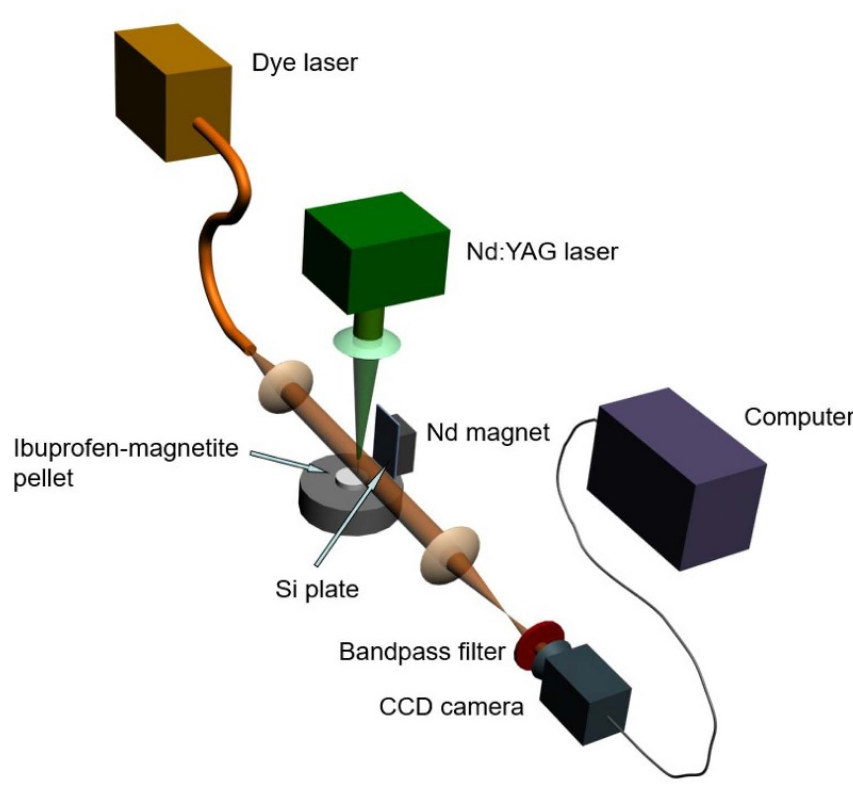

Figure 2. Pump and probe setup for fast photography measurements.

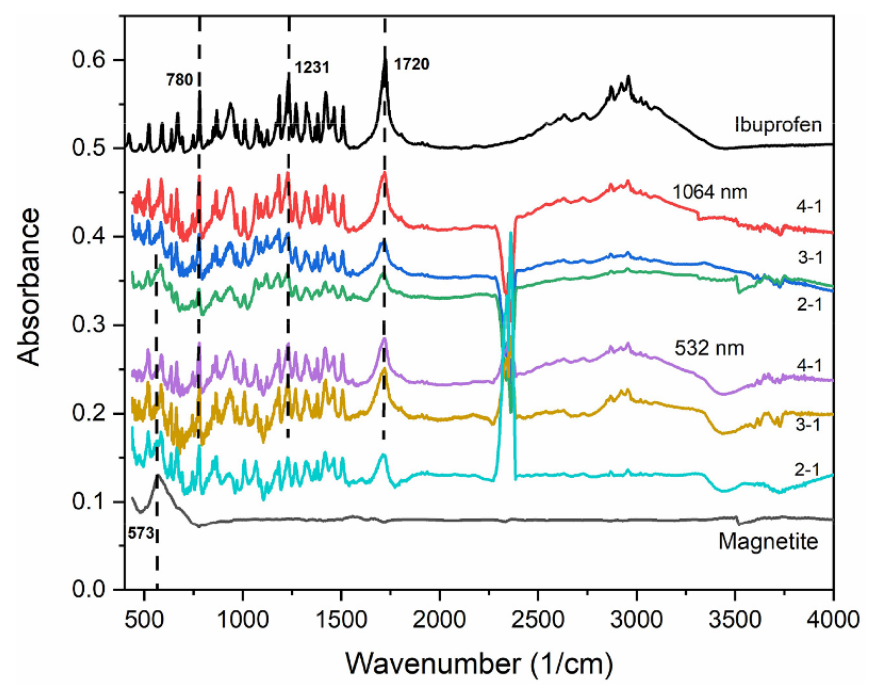

Figure 3. FTIR spectra of PLA generated particles collected on the membrane filters. The laser wavelengths $(1064 \mathrm{~nm}, 532 \mathrm{~nm})$ and the mass ratios of ibuprofen:magnetite $(4-1,3-1,2-1)$ in the targets are indicated.

of $9 \mathrm{~J} \mathrm{~cm}^{-2}$. The fluence was carefully selected not to destroy the target material but still to gain sufficient ablation yield for analysis [23].

Figure 1 shows our basic experimental setup. The tablets were fixed on a rotating sample holder at the bottom of the reaction chamber. We focused the laser beam on the target surface at a grazing angle of $45^{\circ}$ by an N-BK7 fused silica plano convex lens (focal length $=17.5 \mathrm{~cm}$ ). A constant flow of $\mathrm{N}_{2}$ gas (flow rate: $0.3-21 \mathrm{~min}^{-1}$ ) through the chamber provided a non-oxidative environment and directed the ablated particles towards the outlet. At the outlet, the particles were either collected on a membrane filter (pore size $\sim 1 \mu \mathrm{m}$, Merck Millipore Ltd Omnipore Membrane Filter) for chemical analysis, or directed straight into the particle size analyzer.

\subsection{Chemical composition characterization methods}

To ensure that the chemical composition of the ablated products is identical with that of the initial compounds, the ablated particles were analyzed by Fourier-transform infrared (FTIR) and Raman spectroscopy.

3.2.1. FTIR spectroscopy. We analyzed the ablated particles depositing on the membrane filters as well as those which were directed sideways from the ablation plume by a magnet and were collected on silicon plates (see figures 1 and 2). Particles were scraped from the filters/Si plates and mixed with $\mathrm{KBr}$ powder for pellet formation. FTIR spectra were recorded by a Thermo Nicolet AVATAR 330 Spectrometer (LabX Midland, ON, Canada) in the $4000-400 \mathrm{~cm}^{-1}$ range, at a resolution of $4 \mathrm{~cm}^{-1}$, using the average of 128 scans.

3.2.2. Raman spectroscopy. Raman spectroscopy measurements were carried out by a Thermo Scientific, DXR Raman microscope. A laser beam of $\lambda=532 \mathrm{~nm}$ wavelength and $0.1-2 \mathrm{~mW}$ power was used with a $0.7 \mu \mathrm{m}$ spot size on the sample. We applied a $50 \mu \mathrm{m}$ slit spectrograph aperture and 900 lines $\mathrm{mm}^{-1}$ grating. Raman spectra were recorded in the $200-1800 \mathrm{~cm}^{-1}$ range with a resolution of $5.4 \mathrm{~cm}^{-1}$. All spectra were recorded by scanning ten times with a $4 \mathrm{~s}$ integration time. Spectra were recorded from several places of the samples covering the membrane filters to obtain information about their heterogeneity.

\subsection{Particle size analysis with a scanning mobility particle sizer (SMPS)}

We measured the particle size distribution in the $10-800 \mathrm{~nm}$ range with SMPS (TSI system Model 3938, USA). We set the scanning time to $5 \mathrm{~min}$ and repeated the measurements at least three times. The gas flow was $0.31 \mathrm{~min}^{-1}$ during the scans. Because the tablets wear away during the relatively long scanning time, here we lowered the laser pulse repetition rate to $0.3 \mathrm{~Hz}$ during ablation.

\subsection{SEM and energy dispersive $x$-ray spectroscopy (EDX)}

The morphology and elemental composition of the ablated particles were studied by SEM (Hitachi S-4700 FESEM) coupled with EDX spectroscopy (RÖNTEC XFlash Detector). For SEM and eventual transmission electron spectroscopy (TEM) analyses, the particles were placed on TEM-grids by passing the carrier gas stream through the grids at the outlet.

\subsection{Fast photography}

We set up a common pump and probe system (figure 2) to visually observe the ablation process and the effect of the magnetic field on the trajectory of PLA generated particles. The pump 

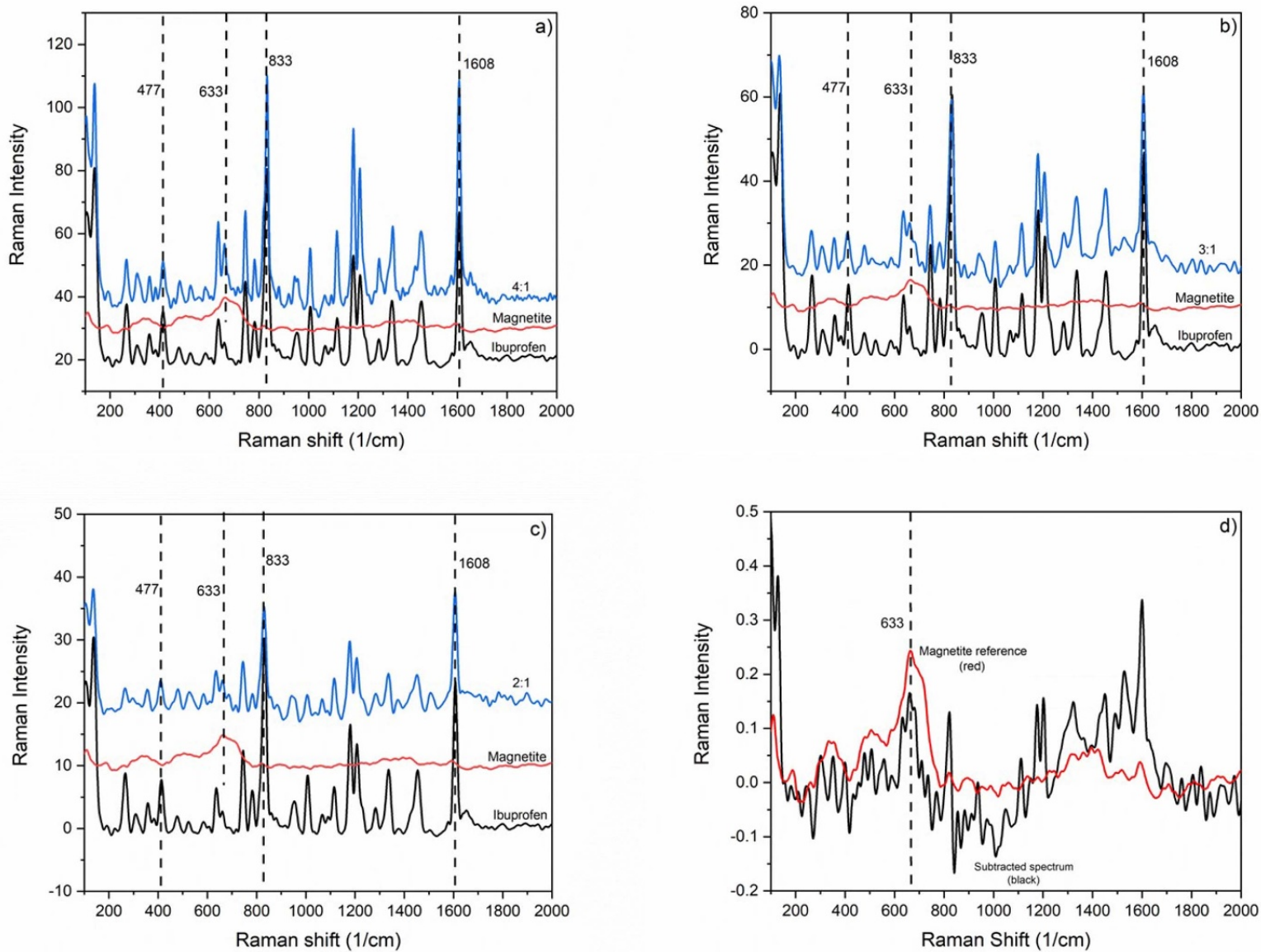

Figure 4. Raman spectra of PLA ( $\lambda=532 \mathrm{~nm}$ ) generated particles (blue). Ibuprofen:magnetite mass ratios in the blended targets were (a) 4:1; (b) 3:1 and (c) 2:1. Magnetite (red) and ibuprofen (black) reference spectra are also shown in (a), (b) and (c). Subset (d) shows the magnetite reference spectrum (red) and the magnetite spectrum obtained by subtraction (see text for details) in case of a blended target sample with an ibuprofen:magnetite mass ratio of 3:1 (black).

laser was the same Nd:YAG laser (Quantel, FWHM = $6 \mathrm{~ns}$, $\lambda=532 \mathrm{~nm}$ ) as for the PLA experiments. As a probe laser, we built a nitrogen laser induced dye laser adapted to the ablating wavelength. The laser active dye was Rhodamine 6G $(\lambda=590 \mathrm{~nm}$, FWHM $=1 \mathrm{~ns})$ in order to avoid an overlap between the pump and probe signals. The dye laser beam was transported to the experimental region by an optical fiber and collimated with an N-BK7 focusing lens $(f=17.5 \mathrm{~cm})$. A CCD camera (The Imaging Source-DMK 23G445, $30 \mathrm{fps}$, $N=5 \times$ ) was placed opposite the optical fiber. We applied a bandpass optical filter in front of the camera to filter out the white light of the generated plasma. The controlling element of this setup was the digital delay generator (SRS-DG645), which can separately trigger the pump and the probe lasers and the camera with a delay time in the nanosecond to millisecond range.

\section{Results}

\subsection{FTIR spectroscopy of the PLA generated particles}

The FTIR spectra of the particles collected on the membrane filters are shown in figure 3. The characteristic peaks of ibuprofen (e.g. $1720 \mathrm{~cm}^{-1}$ (H-bonded $\left.\mathrm{C}=\mathrm{O}\right) ; 1231 \mathrm{~cm}^{-1}(\mathrm{H}$ bonded $\left.\mathrm{CO}-\mathrm{H}) ; 780 \mathrm{~cm}^{-1}(\mathrm{C}=\mathrm{O})\right)$ [27] and magnetite nanopowder $\left(573 \mathrm{~cm}^{-1}\right)$ [28] can be identified in each spectrum, indicating that the ablated particles consist of the chemically unchanged initial compounds. Two peaks between $2250 \mathrm{~cm}^{-1}$ and $2500 \mathrm{~cm}^{-1}$ are related to the $\mathrm{H}_{2} \mathrm{O}$ and $\mathrm{CO}_{2}$ content of the samples.

(We note, that any negative peaks in the $2250-2500 \mathrm{~cm}^{-1}$ range are only due to the different water content of the samples and the clean filter used for background subtraction.)

\subsection{Raman spectroscopy of the PLA generated particle}

The investigation of the ibuprofen-magnetite composite particles by Raman spectroscopy was challenging since the optimal excitation laser power required for spectrum recording differed significantly for the two compounds.

The Raman spectra of particles produced by PLA at $532 \mathrm{~nm}$ and $1064 \mathrm{~nm}$ laser beam wavelengths are shown in figures 4 and 5, respectively. The characteristic ibuprofen peaks (477, 833 and $1608 \mathrm{~cm}^{-1}$ ) [28] are clearly present in these spectra. However, the identification of magnetite by Raman spectroscopy is problematic since magnetite is susceptible to the photolytic and pyrolytic effects of the excitation laser light, 

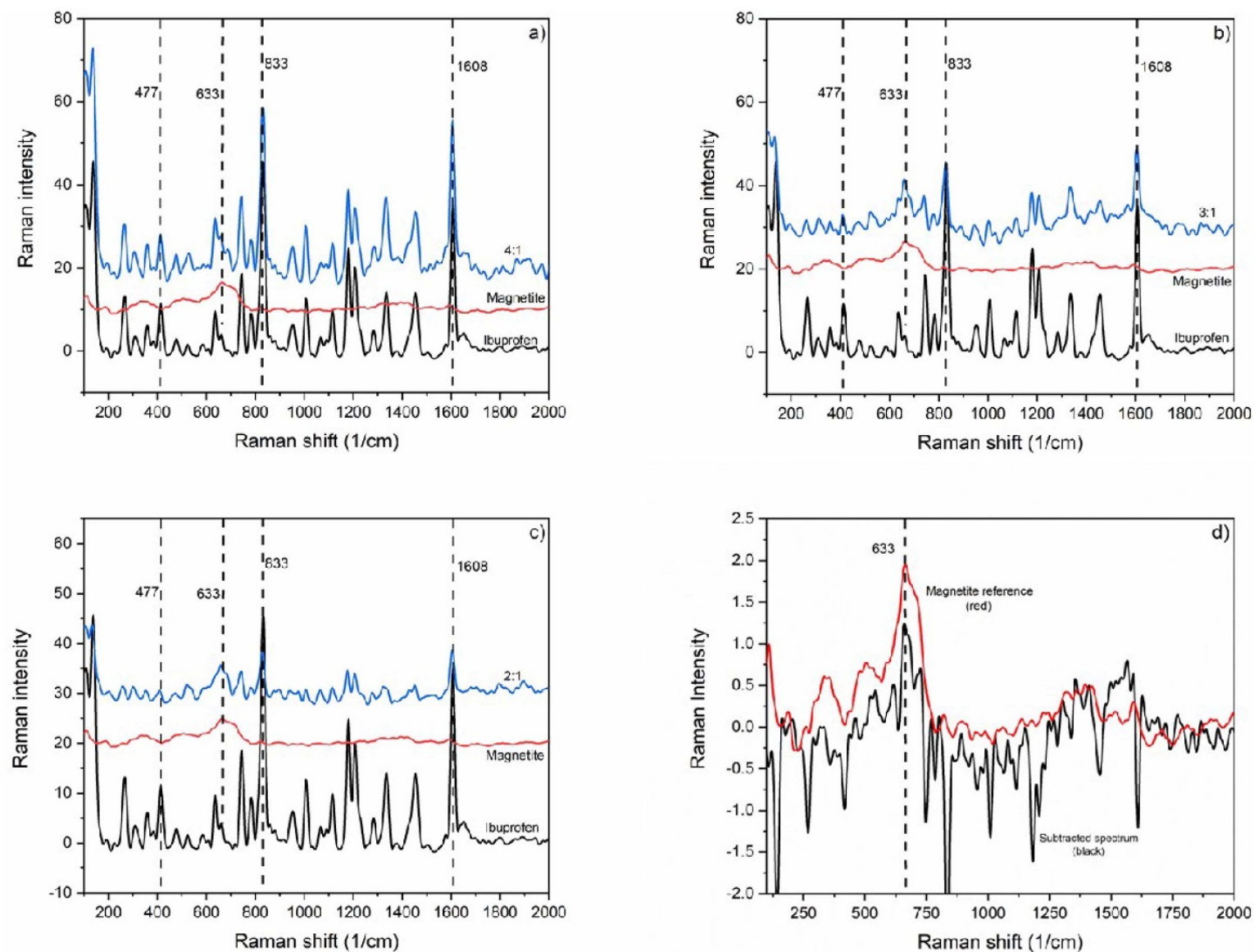

Figure 5. Raman spectra of PLA $(\lambda=1064 \mathrm{~nm})$ generated particles (blue). Ibuprofen:magnetite mass ratios in the blended targets were (a) 4:1; (b) 3:1 and (c) 2:1. Subset (d) shows the magnetite reference spectrum (red) and magnetite spectrum obtained by subtraction (see text for details) in case of a blended target sample with an ibuprofen:magnetite mass ratio of 3:1 (black).

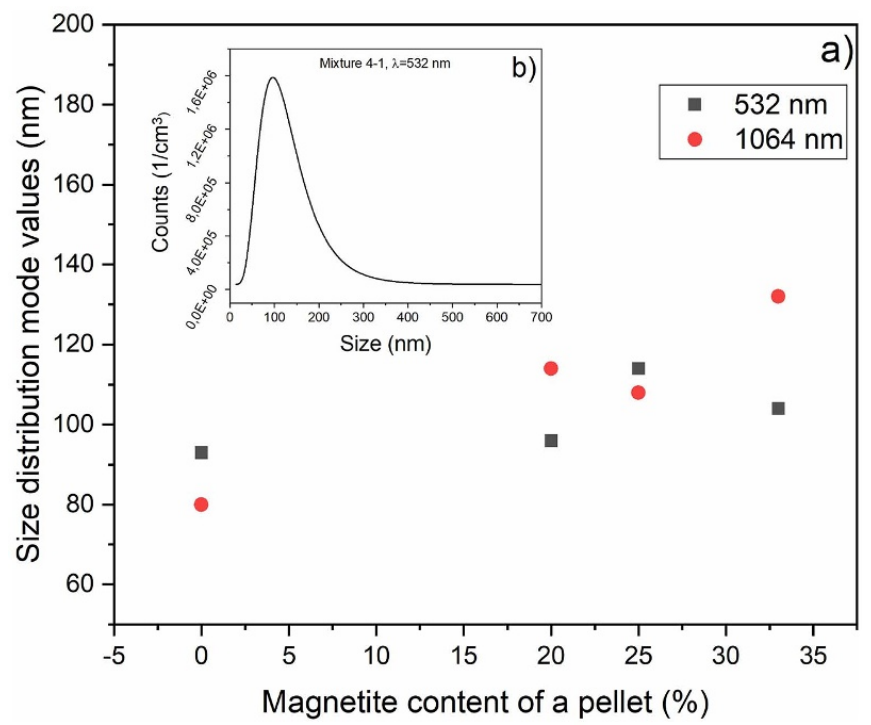

Figure 6. Results of the SMPS measurements: (a) size distribution mode values for various ibuprofen:magnetite ratios; (b) a typical particle size distribution of ablated particles.

and spectral changes may occur due to sample transformation while recording the Raman spectrum [29]. Therefore, we applied the lowest laser powers which still resulted in valuable spectral details for both components. However, the intensity of the most prominent magnetite Raman peak $\left(663 \mathrm{~cm}^{-1}\right)$ was an order of magnitude lower than the intensity of the ibuprofen peaks. To be able to detect the magnetite content, the reference spectrum of ibuprofen was subtracted from the spectra of the composite particles after normalization for the ibuprofen peak at $833 \mathrm{~cm}^{-1}$ (figures 4(d) and 5(d)). Even though these ibuprofen-free spectra (i.e. those corresponding to magnetite only) are rather dissimilar, the characteristic magnetite peak at $633 \mathrm{~cm}^{-1}$ can be identified for all PLA produced particles.

\subsection{Particle size analysis}

We investigated the size distribution of the PLA generated particles in the $10 \mathrm{~nm}-1 \mu \mathrm{m}$ size range. The low laser pulse repetition rate $(0.3 \mathrm{~Hz})$ introduces uncertainty into the measurements since the particles reached the filter intermittently.

A typical size distribution and the mode values of the PLA generated particles when $532 \mathrm{~nm}$ and $1064 \mathrm{~nm}$ laser wavelengths are used can be seen in figure 6 . The mode values are 90-130 $\mathrm{nm}$ for the composite targets, and 80-90 nm for the pure ibuprofen target, which is in good agreement with our former observation [23]. Without claiming completeness, 


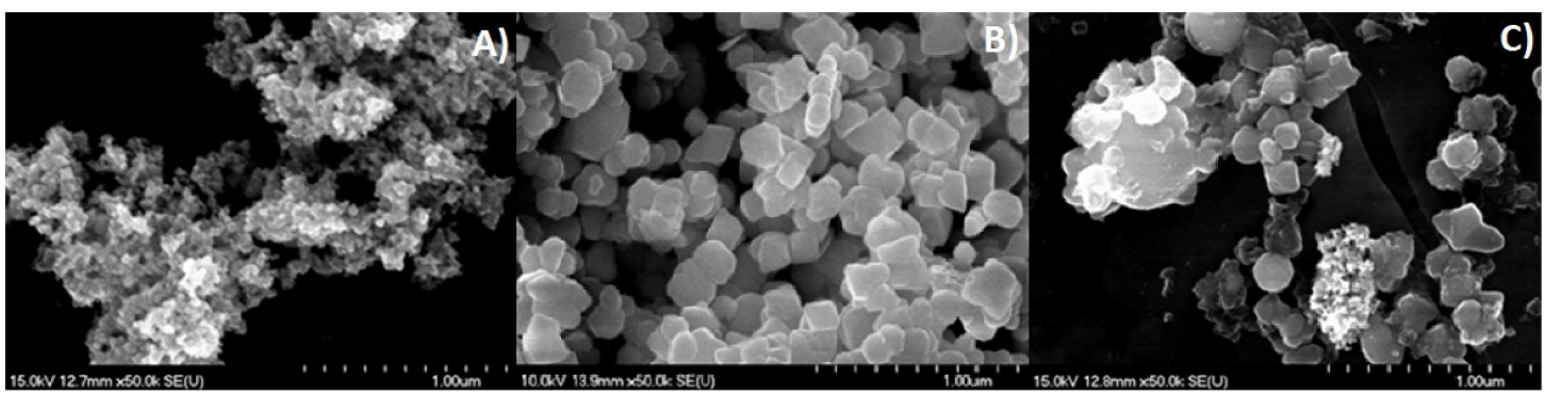

Figure 7. SEM images of PLA generated particles from (a) pure ibuprofen tablet target; (b) pure magnetite nano-powder target and (c) composite ibuprofen:magnetite tablet target with a mass ratio of 3:1. Magnification: $50000 \times$.

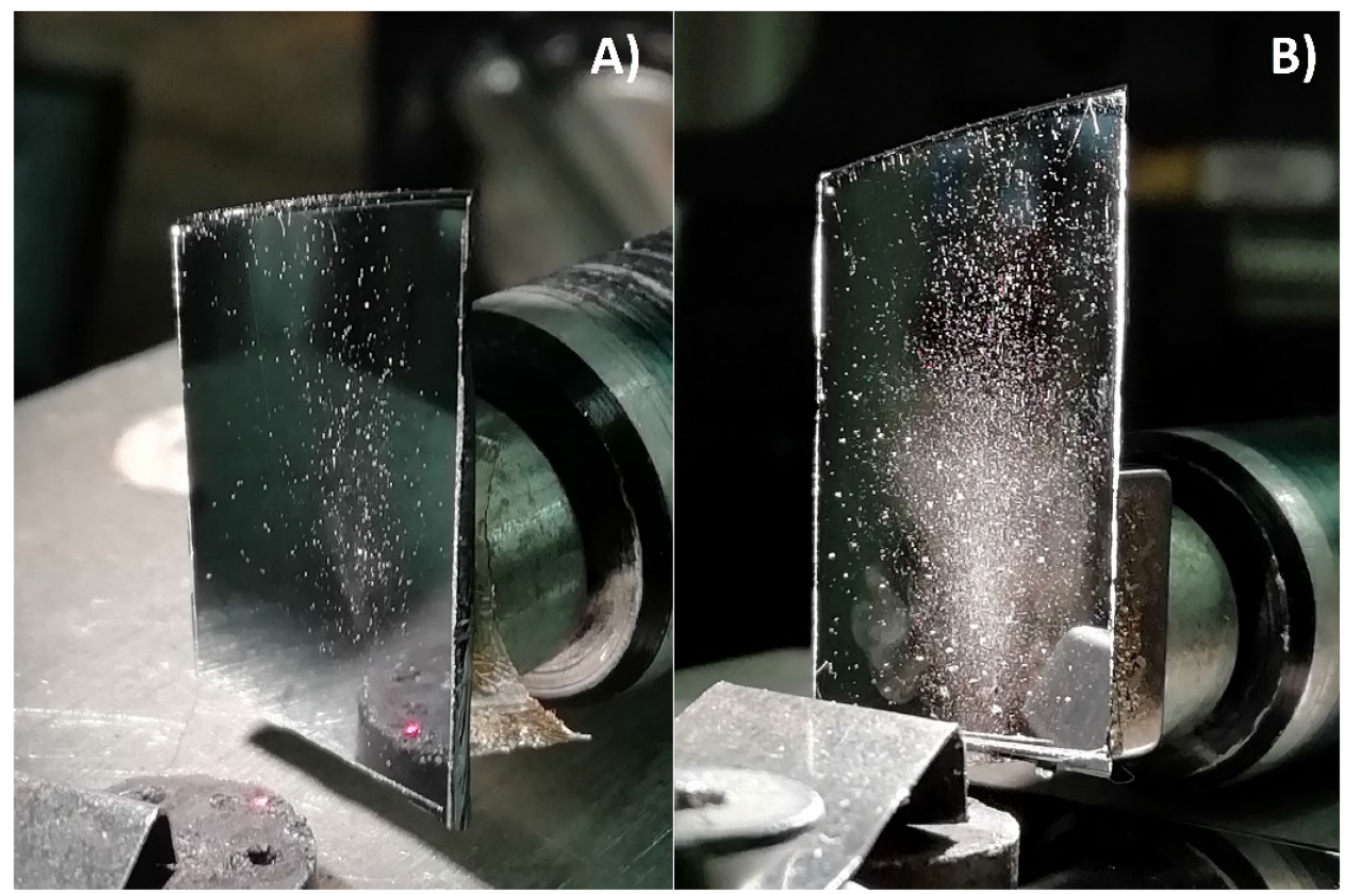

Figure 8. PLA generated particles deposited onto Si plate (a) in the absence of a magnetic field (b) in the presence of a magnetic field.

it must be noted that the maximum transportable particle size is limited by the gas flow rate, which cannot be more than $0.31 \mathrm{~min}^{-1}$ for SMPS.

\subsection{SEM investigation of PLA generated particles}

Figure 7 shows the SEM images of ablated pure ibuprofen, magnetite and composite particles collected on membrane filters. The magnetite and ibuprofen parts of the composite particles can be distinguished to some extent based on their morphological differences. The occurrence of larger particles $(>150 \mathrm{~nm})$ on the filters as compared to the particle sizes measured by SMPS (80$130 \mathrm{~nm})$, can semi-quantitatively be explained by the higher $\left(21 \mathrm{~min}^{-1}\right)$ gas flow rate applied during collection, which has increased the upper size limit of transportable particles. Moreover, generated particles can condensate on the filter too.

\subsection{Fast photography in the absence and presence of a magnetic field}

We used fast photography to demonstrate the effect of magnetic field on the ablated products and to verify the magnetite content of the particles.

Figure 8 shows the particle abundance deposited onto $\mathrm{Si}$ plates placed sideways from the ablation plume and having (figure 8(b)) or not having (figure 8(a)) a magnet behind. It can be clearly seen that the number of deposited particles are magnitudes higher when a magnetic field is applied.

Figure 9 shows that in the absence of a magnetic field the particles are ejected in the direction normal to the substrate surface (figure 9(a)). However, the trajectory of the ablated particles can be significantly modified by a magnet placed sideways (figure 9(b)). The influence of the magnetic deflecting force is more noticeable in the case of smaller particles, which could either indicate their higher magnetite mass content, or it could simply be the consequence of their lower mass 


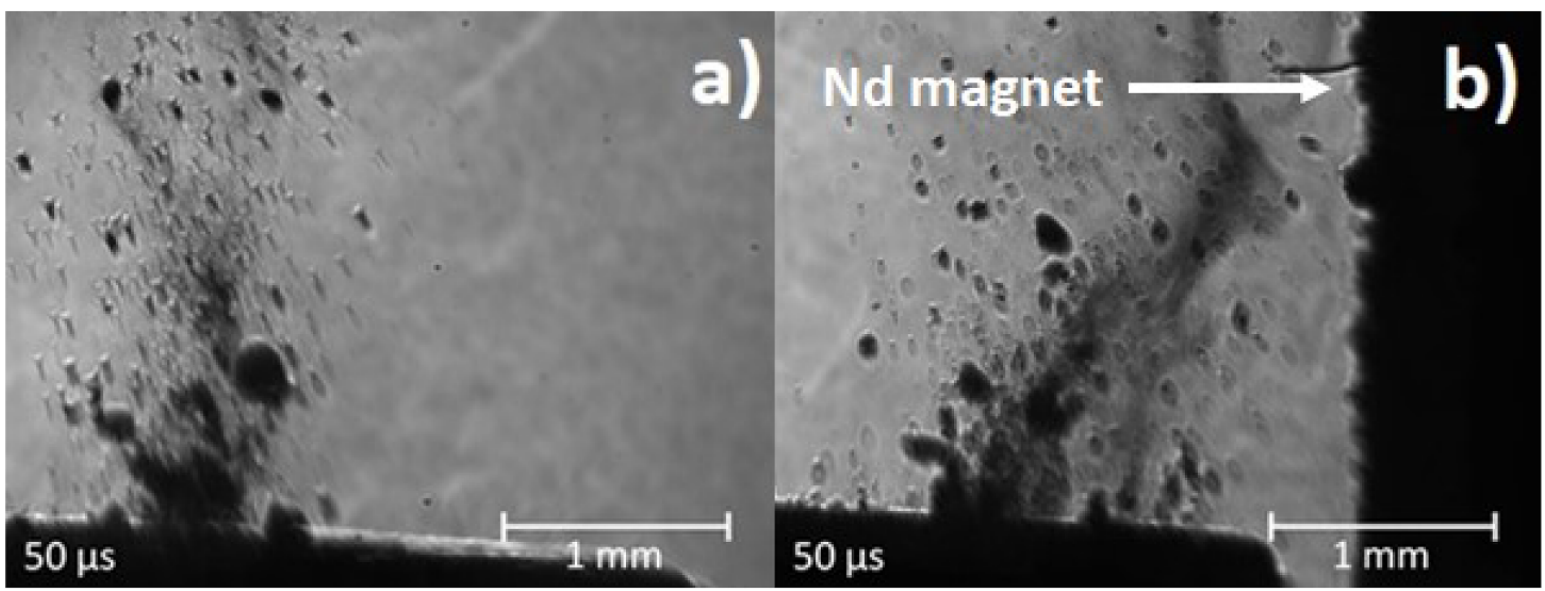

Figure 9. Fast photography images of ejected particles generated by the PLA of ibuprofen-magnetite composite tablets (a) without any external magnetic field; (b) in the presence of a magnetic field.

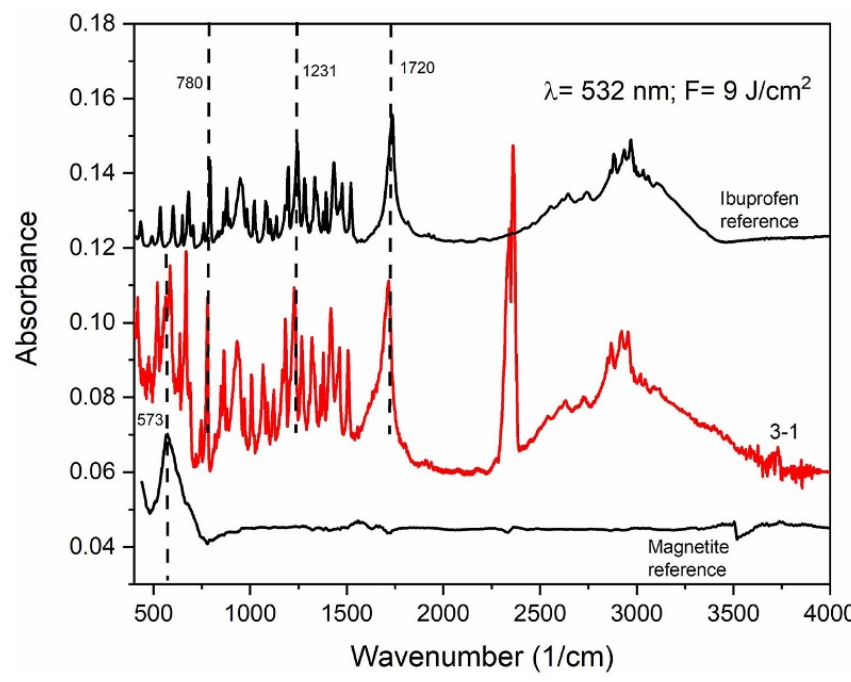

Figure 10. FTIR spectra of PLA generated particles collected on the silicon plate placed sideways (with a magnet behind) at $532 \mathrm{~nm}$ laser wavelength in case of an ibuprofen:magnetite mass ratio of 3:1.

even if they contain the same amount (and up to a point, even lower amount) of magnetite as the heavier ones.

\subsection{Results in the presence of a magnetic field}

4.6.1. FTIR spectroscopy. Figure 10 shows the FTIR spectrum of particles being collected on the Si plate. We display the characteristic bands of ibuprofen (e.g. $1720 \mathrm{~cm}^{-1}$ (H-bonded $\mathrm{C}=\mathrm{O}) ; 1231 \mathrm{~cm}^{-1}$ (H-bonded CO-H); $\left.780 \mathrm{~cm}^{-1}(\mathrm{C}=\mathrm{O})\right)$ [27] and magnetite $\left(573 \mathrm{~cm}^{-1}\right)$ [30]. Here we present only one spectrum of the ablated particles using a composite target with an ibuprofen:magnetite mass ratio of $3: 1$. The presence of the characteristic peaks of both compounds indicates the composite character of the ablated particles.

\subsubsection{SEM and EDX investigation of PLA generated particles.}

Figure 11 depicts the SEM image, the elemental composition map and the EDX spectrum taken from the same particles.
These particles were directed onto the collecting silicon plate by a magnetic field. The overlapping carbon (C) and iron (Fe) content of the particles indicates their composite character.

\subsubsection{Particle size measurement (SMPS) in the presence of} a magnetic field. We made another modification in our first experimental setup for particle size measurement. To measure the size distribution of the magnetite containing (i.e. magnetic) particles, we put a y-branch in front of the entrance of the SMPS. One of the pipes was connected to the particle sizer (SMPS) and the other pipe was inserted into a vacuum tube where the gas flow was set to the same rate as that of the SMPS $\left(0.31 \mathrm{~min}^{-1}\right)$. With the help of an Nd magnet, the magnetic particles were guided into the pipe connected to the SMPS. The size distribution of the deflected magnetic particles is shown in figure 12. Due to the uncertainty in the ablation yield, we repeated the measurements several times (with and without using the magnet). In figure 12 we represent the averaged size distribution curves. It can be seen that the detected portion of the ablation yield grows with magnetic field assistance.

\section{Discussion}

In the course of PLA, two main processes must be considered. At first, the focused laser beam induces a microexplosion in the target. The maximum temperature depends on the optical absorption coefficient $(\alpha)$, the specific heat $(c)$ and the density $(\rho)$ of the material, as well as on the applied fluence of the laser beam. At this stage, vaporized and strongly decomposed products are formed and ejected from the surface. Secondly, the high temperature and pressure conditions trigger the formation of shock waves and recoil forces in the bulk. The photomechanical effects eventually induce the ejection of chemically preserved material. When interpreting the ablation mechanism of our targets, we must consider the following: (a) our targets are porous, which results in lower temperatures, higher photomechanical effects and higher ablation yields [23] compared to the bulk materials. (b) The targets are 

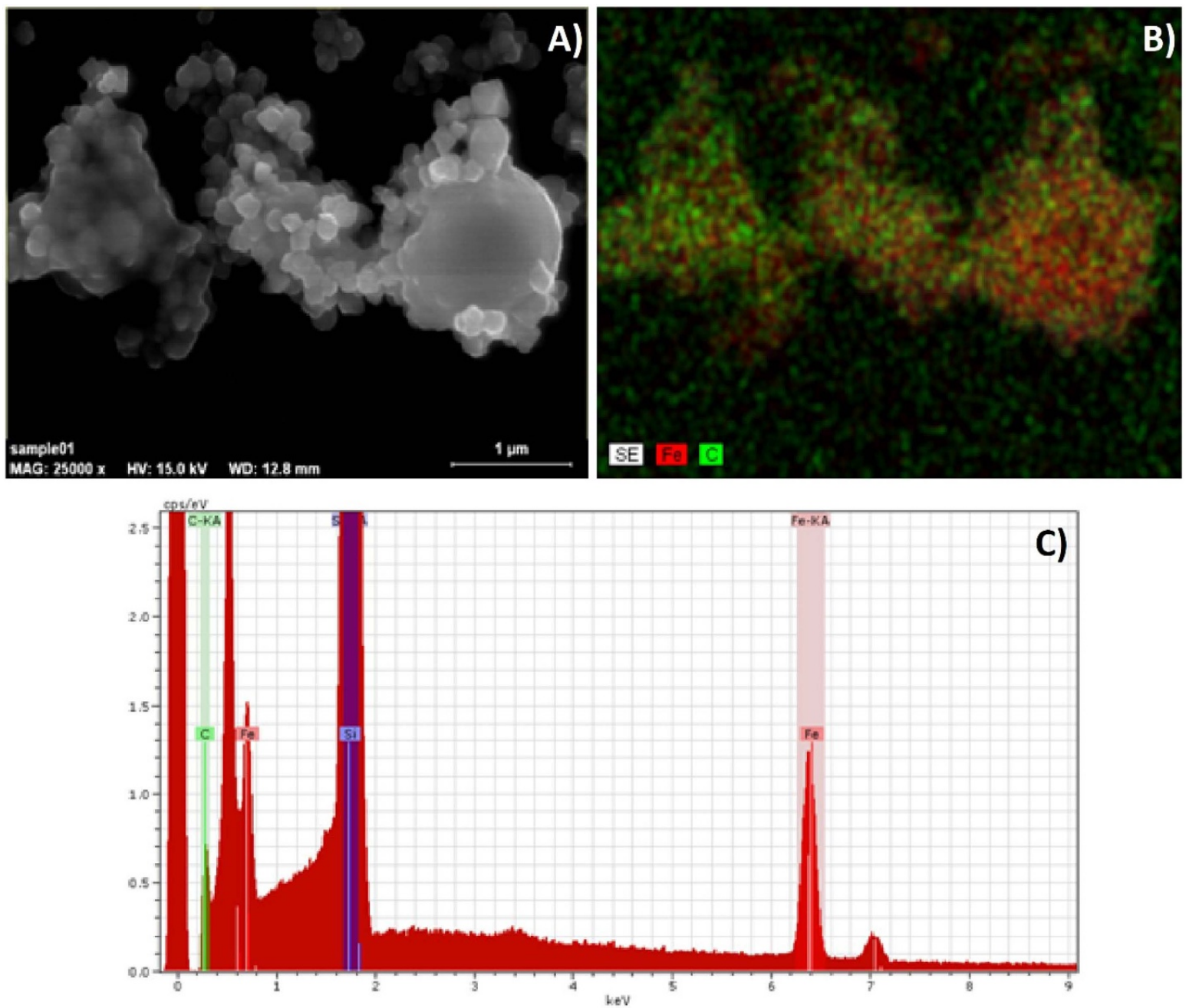

Figure 11. (A) SEM image of ablated particles being collected on a Si plate by a magnetic field. The ibuprofen: magnetite mass ratio in the target tablet was 3:1, and the applied laser wavelength was $\lambda=532 \mathrm{~nm}$. (B) EDX element mapping of the same area. The green and red dots correspond to carbon $(\mathrm{C})$ and iron $(\mathrm{Fe})$ atoms, respectively. (C) EDX spectrum recorded from the same area.

inhomogeneous both in particle size and optical properties as they are mixtures of two very different materials pressed into pellets. These composite targets show some similarities with the matrix-assisted pulsed laser evaporation (MAPLE) targets. In MAPLE, the solvent absorbs most of the laser energy, evaporates and entrains the solute (usually an organic compound) in intact form. We think that a similar scenario occurs in our case too [31].

To elucidate the ablation mechanism, we made approximate calculations - in accordance with our former study [23]— to determine the temperature rise in the composite targets and the penetration depth $(d)$ of the laser beam. For the calculations we used the physical and thermal properties of bulk ibuprofen and magnetite, since the initial size of the particles fell in the $500-1000 \mathrm{~nm}$ range for ibuprofen and in the 50-100 nm range for magnetite. We used the Beer-Lambert law (equation (1)) to calculate the temperature change (caused by optical heating) at the target surface and at different depths from the surface:

$$
\Delta T(x)=\frac{\alpha \times F}{\rho \times c} \times e^{-\alpha x}
$$

where $\alpha$ is the wavelength dependent absorption coefficient, $x$ is the distance from the surface, $F$ is the applied fluence of the laser beam, $\rho$ is the density of the target and $c$ is the specific heat of the target material. Pure ibuprofen has relatively low optical absorption $\left(\alpha=2 \times 10^{5} \mathrm{~m}^{-1}\right)$ at the applied laser wavelengths, which causes lower maximum temperatures $\left(10^{4} \mathrm{~K}\right)$ and larger penetration depths $\left(d=\alpha^{-1}\right)(3-5 \mu \mathrm{m})$ compared to magnetite. The optical absorption of magnetite is two orders of magnitude higher $\left(\alpha=2 \times 10^{7} \mathrm{~m}^{-1}\right)$ at both applied wavelengths [32], which generates higher temperatures $\left(10^{5} \mathrm{~K}\right)$ at the surface, but smaller penetration depths $(50-100 \mathrm{~nm})$. In the bulk not only the optical heating must be considered, but the heat transfer effects too. For this purpose, we applied the linear heat transfer equation (equation (2)):

$$
\frac{\mathrm{d} Q}{\mathrm{~d} t}=-\lambda \times A \times \frac{\mathrm{d} T}{\mathrm{~d} x}
$$

where $\mathrm{d} Q / \mathrm{d} t$ is the rate of heat transfer, $A$ is the area of cross-section of the heat flow path, $\lambda$ is the heat conductivity of the material, $\mathrm{d} T / \mathrm{d} x$ is the temperature gradient. 


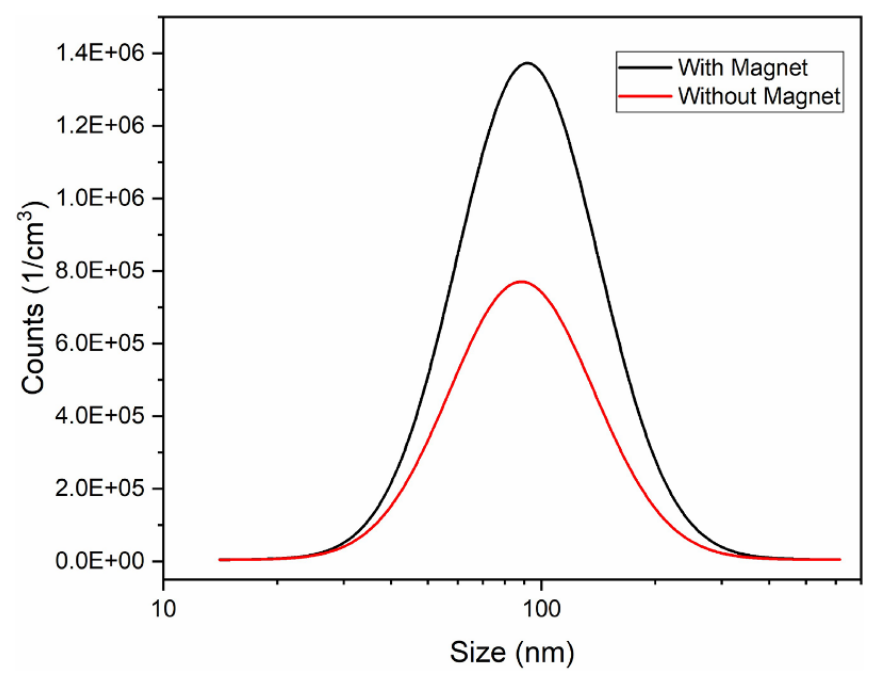

Figure 12. Particle size distribution of the generated particles using $532 \mathrm{~nm}$ laser wavelength and $9 \mathrm{~J} \mathrm{~cm}^{-2}$ fluence in case of using a deflecting magnet (black) and without a magnet (red).

We must consider different scenarios, depending on the uppermost surface structure of the target at the site of the laser shot. (a) When the laser beam is incident on a relatively large $(10-15 \mu \mathrm{m})$ ibuprofen particle, most of the laser energy is absorbed by the ibuprofen, and the adjacent magnetite particles remain intact. Here photomechanical effects induce mainly particle fracturing [23]. (b) When magnetite NPs, which are approximately equal in size with the penetration depth, are irradiated directly by the laser beam at the target surface, the ablation process depends on the depth of embedding of the ibuprofen particles. The SEM images reveal that the ibuprofen particles are typically surrounded and covered by some layers of magnetite NPs. First, let us consider a $200 \mathrm{~nm}$ thick magnetite coverage (approximately two layers of NPs) over the ibuprofen particle. In this case, due to the optical absorption of magnetite, (using equation (1)), a $\sim 9 \times 10^{3} \mathrm{~K}$ temperature rise at the surface of the embedded ibuprofen particle must be taken into account. (The temperature rise from transmitted laser fluence is by orders of magnitude smaller, and therefore negligible.) Using an approximate heat conductivity $\left(\lambda=0.1 \mathrm{Wm}^{-2} \mathrm{~K}^{-1}\right)$ for ibuprofen, which can be a good estimate for an organic compound, we calculated that a relatively thin $(\sim 150 \mathrm{~nm})$ upper layer of ibuprofen decomposes $\left(T_{\text {dec.ibu }}=250{ }^{\circ} \mathrm{C}\right)$ down to $350 \mathrm{~nm}$ under the tablet surface, while it evaporates down to $2.35 \mu \mathrm{m}\left(T_{\text {boilibu }}=157^{\circ} \mathrm{C}\right)$ and melts down to a depth of $7 \mu \mathrm{m}\left(T_{\text {melt.ibu }}=75^{\circ} \mathrm{C}\right)$ (figure 13(A/I)). The molten or vaporized ibuprofen can condense on the magnetite NPs (figure 13(B)) resulting in the formations observed by SEM. Now, if we consider that the laser beam hits a region containing only magnetite adjacent to the ibuprofen (figure 13(A/II)), then the ablation of the top $300 \mathrm{~nm}$ magnetite layer can be described by the optical absorption model. This is the depth above which the temperature rise (due to optical heating) exceeds the melting point of magnetite. Below this depth, heat conduction of solid magnetite is the main heat transfer mechanism, resulting in elevated temperatures in the target bulk. Calculating with the significantly higher heat conductivity of bulk magnetite $\left(\lambda=3.5 \mathrm{Wm}^{-2} \mathrm{~K}^{-1}\right)$ we obtained that the temperature can exceed $T_{\text {dec.ibu }}$ even at $2.46 \mu \mathrm{m}$ below the target surface in the region containing only magnetite. Thus, even those Ibuprofen particles (or particle parts) which are embedded $2.46 \mu \mathrm{m}$ deep in the target can decompose, evaporate or melt due to the heat transfer from the nearby magnetite in adjacent regions. This means that the magnetite particles surrounding the ibuprofen particles provide additional heat (the intensity of which decreases with depth) from the lateral side of the ibuprofen particles (figure 13(A)). We must note that our targets are porous, and the above calculations give a strong upper estimation for the temperatures and characteristic depths of ablation. According to our SEM-EDX investigations, unmerged magnetite and ibuprofen particles are also generated during the ablation. Figure 13 illustrates a schematic approach to particle production, where composites are formed in a pure core-shell structure. Considering the chaotic particle motions during ablation, as well as the sizes and morphologies observed in SEM images, it is clear that the merged particles have a variety of morphologies, such as matrix dispersed, Janus or even inverse core-shell structure [33].

As can be seen in figure 9, particles with a wide size distribution are produced during ablation. The biggest and heaviest particles immediately fall down before reaching the membrane filter or the entrance of SMPS. According to the FTIR and Raman spectroscopy investigations (figures 3-5), the generated particles contain chemically preserved ibuprofen and magnetite NPs in case of both applied wavelengths. The presence of intact ibuprofen (showing no sign of molecular decomposition) indicates that these products must originate from the secondary, photomechanical ablation process. The results of particle size analysis (figure 6) are in good agreement with our former studies [23] and with the initial particle size of magnetite. We cannot see any clear tendencies in particle size distributions in terms of wavelengths or different mass ratios of the constituents. This observation is most likely related to the porosity of the targets, which leads to uncertainties in the determination of the ablation yield and the mode values of the size distributions too. PLA generated pure ibuprofen and pure magnetite particles look quite different and can easily be distinguished in the SEM images (figure 7).

While searching for more evidence for the merge of ibuprofen and magnetite NPs we also studied the particles behavior in an external magnetic field. We found that small particles could be easily diverted by the magnetic field, but larger and heavier particles simply kept moving in their initial direction (figure 9). This behavior of the large particles is related to their lower specific magnetism as they are typically larger ibuprofen particles with only a few magnetite NPs stuck to their surface. EDS analysis of the diverted particles has shown that in addition to magnetite, these products have a relatively high ibuprofen content (figures 10 and 11), which is a further proof of merging of the two components. 


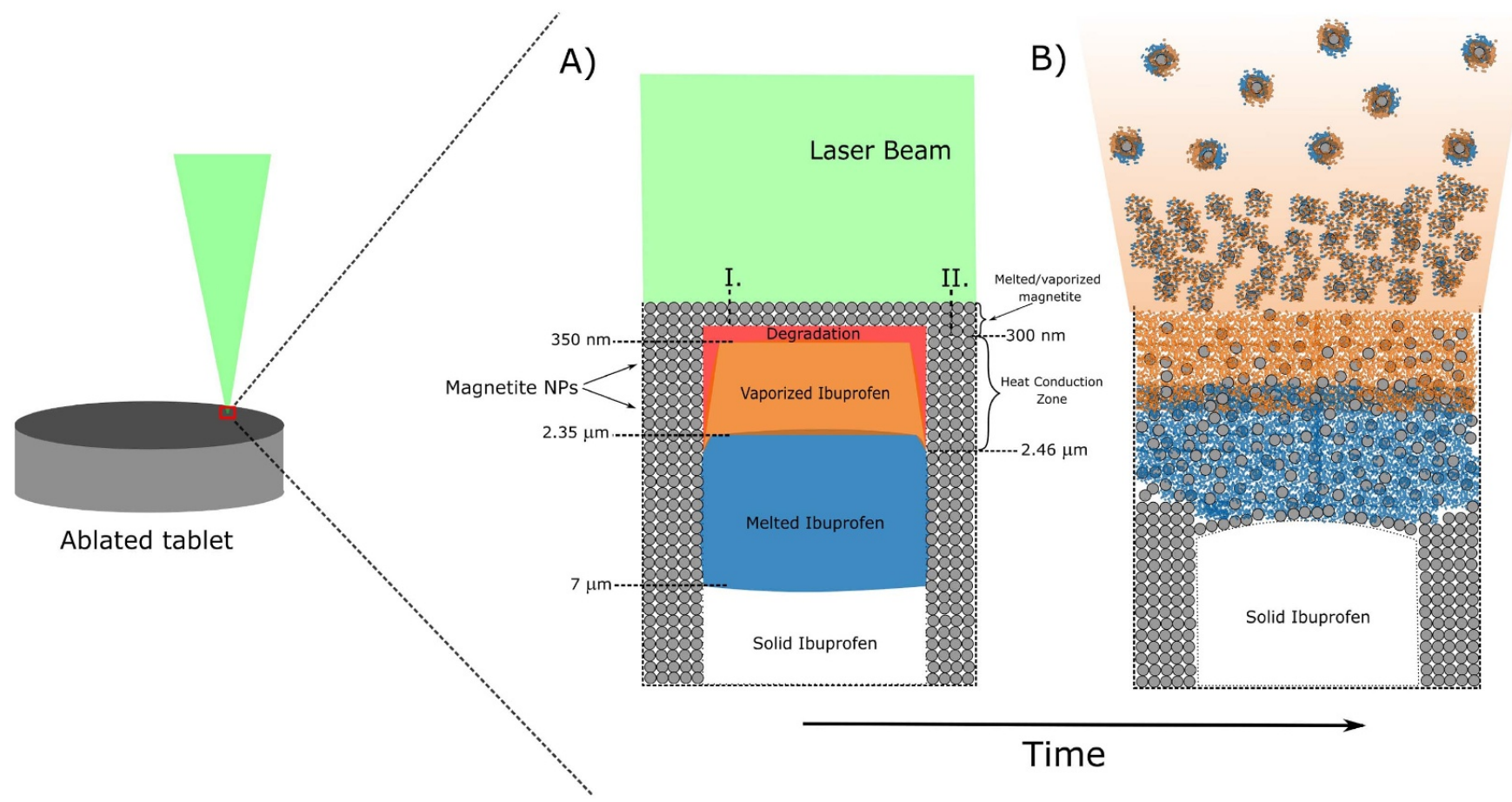

Figure 13. Schematic layout of (A) laser-irradiated volume of ibuprofen-magnetite tablets; (B) composite nanoparticle evolution during the explosion of PLA.

\section{Conclusion}

We have successfully produced ibuprofen-magnetite composite NPs by PLA. The size of the particles was in the 100$150 \mathrm{~nm}$ range and depended both on the mass ratio of the ingredients in the target pellet and on the laser wavelength. We confirmed the magnetite content of the particles and the preserved chemical character of the drug constituent. Our simple model calculations suggest that the high absorption coefficient of magnetite results in the melting and evaporation of ibuprofen during ablation, and that the composite particles are formed by the condensation or merging of ibuprofen on the surface of magnetite NPs.

PLA can provide a simple and chemical-free method for making composite NPs from a pharmaceutically active compound and MNPs. This approach can be used for the development of new drug formulations, especially for targeted drug delivery applications.

\section{Data availability statement}

All data that support the findings of this study are included within the article (and any supplementary files).

\section{Acknowledgments}

This work was supported by the GINOP-2.3.2-15-201600036 Grant ('Development and application of multimodal optical nanoscopy methods in life and materials sciences') and the Ministry of Human Capacities, Hungary Grant (TUDFO/47138-1/2019-ITM FIKP program). T Ajtai is indebted for his Bolyai János Research Scholarship of the Hungarian Academy of Sciences.

\section{ORCID iDs}

Tamás Gera (D) https://orcid.org/0000-0002-0642-0423

Zoltán Bozóki (D) https://orcid.org/0000-0003-3638-9524

Béla Hopp (D) https://orcid.org/0000-0002-4272-7721

\section{References}

[1] Abu-Dief A M and Abdel-Mawgoud A A H 2018 Functionalization of magnetic nanoparticles for drug delivery SF J. Nanochem. Nanotechnol. 11005

[2] Din Ud F, Aman W, Ullah I, Qureshi O S, Mustapha O, Shafique S and Zeb A 2017 Effective use of nanocarriers as drug delivery systems for the treatment of selected tumors Int. J. Nanomed. 12 7291-309

[3] Javadzadeh Y and Azharshekoufeh Bahari L 2017 Therapeutic nanostructures for dermal and transdermal drug delivery Nano- and Microscale Drug Delivery Systems (Amsterdam: Elsevier) pp 131-46

[4] Chamundeeswari M, Jeslin J and Verma M L 2019 Nanocarriers for drug delivery applications Environ. Chem. Lett. 17 849-65

[5] Thierry B 2009 Drug nanocarriers and functional nanoparticles: applications in cancer therapy Curr. Drug Delivery 6 391-403

[6] Yestisgin Alp, Cetinel Sibel, Zuvin Merve and Kosar Ali Therapeutic nanoparticles and their targeted delivery applications Delivery Appl. Delivery Applications (https:// doi.org/10.3390/molecules25092193)

[7] Liu L et al 2015 A new approach to reduce toxicities and to improve bioavailabilities of platinum-containing anti-cancer nanodrugs Sci. Rep. 5 1-11 
[8] Rajabi M, Srinivasan M and Mousa S A 2016 Nanobiomaterials in drug delivery Nanobiomaterials in Drug Delivery (Amsterdam: Elsevier) pp 1-37

[9] Pylypchuk I V, Kołodyńska D, Kozioł M and Gorbyk P P 2016 Gd-DTPA adsorption on chitosan/magnetite nanocomposites Nanoscale Res. Lett. 11168

[10] Price P M, Mahmoud W E, Al-Ghamdi A A and Bronstein L M 2018 Magnetic drug delivery: where the field is going Front. Chem. 6 1-7

[11] Namdeo M, Saxena S, Tankhiwale R, Bajpai M, Mohan Y M and Bajpai S K 2008 Magnetic nanoparticles for drug delivery applications J. Nanosci. Nanotechnol. $83247-71$

[12] Vangijzegem T, Stanicki D and Laurent S 2019 Magnetic iron oxide nanoparticles for drug delivery: applications and characteristics Expert Opin. Drug Delivery 16 69-78

[13] Xiong F, Huang S and Gu N 2018 Magnetic nanoparticles: recent developments in drug delivery system Drug Dev. Ind. Pharm. 44 697-706

[14] Gómez-Sotomayor R, Ahualli S, Viota J L, Rudzka K and Delgado Á V 2015 Iron/magnetite nanoparticles as magnetic delivery systems for antitumor drugs J. Nanosci. Nanotechnol. 15 3507-14

[15] Ganapathe L S, Mohamed M A, Mohamad Yunus R and Berhanuddin D D 2020 Magnetite $\left(\mathrm{Fe}_{3} \mathrm{O}_{4}\right)$ nanoparticles in biomedical application: from synthesis to surface functionalisation Magnetochemistry 668

[16] Bu Y, Cui B, Zhao W and Yang Z 2017 Preparation of multifunctional $\mathrm{Fe}_{3} \mathrm{O}_{4} @ \mathrm{ZnAl}_{2} \mathrm{O}_{4}: \mathrm{Eu}_{3+} @ \mathrm{mSiO}_{2}$-APTES drug-carrier for microwave controlled release of anticancer drugs $R S C A d v .755489-95$

[17] Liu C 2005 A Study of Particle Generation during Laser Ablation with Applications (Berkeley, CA: Lawrence Berkeley National Laboratory (LBNL))

[18] Mele A, Giardini-Guidoni A and Teghil R 1993 Laser ablation of inorganic and organic materials J. Chem. Sci. $105715-33$

[19] Gera T, Smausz T, Kopniczky J, Galbács G, Ambrus R, Szabó-Révész P and Hopp B 2019 Production of ibuprofen in crystalline and amorphous forms by pulsed laser deposition (PLD) Appl. Surf. Sci. 493 359-67

[20] Hopp B et al 2018 Production of meloxicam suspension using pulsed laser ablation in liquid (PLAL) technique J. Phys. D: Appl. Phys. 51165401
[21] Ding W, Sylvestre J-P, Leclair G and Meunier M 2012 Laser fragmentation as an efficient size-reduction method for pulmonary drug discovery: proof-of-concept study of beclomethasone dipropionate Int. J. Theor. Appl. Nanotechnol. 1 99-104

[22] Kimura K, Gibo M, Nerome C, Kura T, Ooshiro S and Tamaki Y 2018 Nanoparticle formation by laser ablation of perylene microcrystals in an aqueous solution of Triton X-100 Chem. Phys. Lett. 691 271-5

[23] Gera T et al 2020 Application of pulsed laser ablation (PLA) for the size reduction of non-steroidal anti-inflammatory drugs (NSAIDs) Sci. Rep. 1015806

[24] Huang J, Li Y, Orza A, Lu Q, Guo P, Wang L, Yang L and Mao H 2016 Magnetic nanoparticle facilitated drug delivery for cancer therapy with targeted and image-guided approaches Adv. Funct. Mater. 26 3818-36

[25] National Center for Biotechnology Information 2020 PubChem compound summary for CID 3672 Ibuprofen

[26] Blaney L 2007 Magnetite $\left(\mathrm{Fe}_{3} \mathrm{O}_{4}\right)$ : properties, synthesis, and applications (Lehigh University) 15 37-42

[27] Vueba M L, Pina M E and Batista de Carvalho L A E 2008 Conformational stability of ibuprofen: assessed by DFT calculations and optical vibrational spectroscopy J. Pharm. Sci. $97845-59$

[28] Raz M, Moztarzadeh F, Hamedani A A, Ashuri M and Tahriri M 2012 Controlled synthesis, characterization and magnetic properties of magnetite $\left(\mathrm{Fe}_{3} \mathrm{O}_{4}\right)$ nanoparticles without surfactant under $\mathrm{N}_{2}$ gas at room temperature Key Eng. Mater. 493-494 746-51

[29] Shebanova O N and Lazor P 2003 Raman study of magnetite $\left(\mathrm{Fe}_{3} \mathrm{O}_{4}\right)$ : laser-induced thermal effects and oxidation $J$. Raman Spectrosc. 34 845-52

[30] Toyos-Rodríguez C et al 2019 A simple and reliable synthesis of superparamagnetic magnetite nanoparticles by thermal decomposition of $\mathrm{Fe}(\mathrm{acac})_{3}$ J. Nanomater. 2019 1-10

[31] Caricato A P and Luches A 2011 Applications of the matrix-assisted pulsed laser evaporation method for the deposition of organic, biological and nanoparticle thin films: a review Appl. Phys. A 105 565-82

[32] Schlegel A, Alvarado S F and Wachter P 1979 Optical properties of magnetite $\left(\mathrm{Fe}_{3} \mathrm{O}_{4}\right)$ J. Phys. C 12 1157-64

[33] Zhu N, Ji H, Yu P, Niu J, Farooq M, Akram M, Udego I, Li H and Niu X 2018 Surface modification of magnetic iron oxide nanoparticles Nanomaterials $\mathbf{8} 810$ 